\title{
Impact of Traditional and Modern Clinics on Diabetes Control in Zanzibar: A Case of Urban West Region- Unguja
}

\author{
Zuhura Saleh Amour $^{1^{*}} \quad$ Assoc. Prof. Nsubuga Haroonah ${ }^{2}$ \\ Dr. Abdalla Ussi Hamad ${ }^{3}$ \\ Dr. Rukkaya Wakif Muhammed ${ }^{4}$ \\ 1.Non Communicable Disease Unit Officer, Ministry of Health Zanzibar. P.O.Box 236, Zanzibar \\ 2.Dean, Faculty of Arts and Social Sciences, Zanzibar University, P.O.Box 2440 Zanzibar \\ 3.Head, Department of Economics, Faculty of Arts and Social Sciences, Zanzibar University P.O.Box 2440, \\ Zanzibar, Tanzania \\ 4.Head, Department of Public Administration, Faculty of Arts and Social Sciences, Zanzibar University P.O.Box \\ 2440, Zanzibar, Tanzania
}

\begin{abstract}
This study aimed at examining the impact of traditional and modern clinics on diabetes management in Urban West Region of Unguja. The sample size for this study consisted of 80 geriatrics diabetes patients. Survey questionnaire was used as a data collection instrument. Descriptive as well as inferential statistics were used to analyse the collected data from relevant respondents. The findings showed that, diabetes medicines are available in the clinics for almost (73.8\%). It was found out that, much of the geriatrics diabetes patients around $(56.3 \%)$ believed in modern rather than traditional medicines. However, both of them were used and have positive impact on diabetes control in the Urban West Region of Unguja - Zanzibar. Finally, the study recommends that, provision of education concerning diabetes should be prioritized. The authorities responsible for the supervision of drugs especially ZFDA and Ministry of Health must conduct more research on the traditional medicines. The medicines used for the treatment of the diabetes administered to the geriatrics diabetes patients must be procured in adequate quantities. School curricula should include several topics of diabetes. National multi-sectorial committees should be formulated to review the policy and regulation of traditional services provision and delivery in Zanzibar.
\end{abstract}

Keywords: Geriatrics, Traditional clinics, Modern clinics and Diabetes Control

DOI: $10.7176 / \mathrm{JHMN} / 82-05$

Publication date: November $30^{\text {th }} 2020$

\section{Introduction}

Diabetes is a chronic (long-lasting) health condition that affects how one's body turns food into energy. Stephanie (2020) defines diabetes as metabolic disease that causes high blood sugar where the hormone insulin moves sugar from the blood into one's cells to be stored or used for energy. In addition, with diabetes, the human body either does not make enough insulin or can not effectively use the insulin it makes. The International Diabetes Federation (IDF) (2020) also defines diabetes as chronic disease that occurs when the pancreas is no longer able to make insulin, or when the body can not make good use of the insulin it produces.

The number of elderly people around the world has been steadily increasing, with projections showing the age group being composed of $22 \%$ of total population by 2050 (Pillemer, et.al., 2016). Non-communicable diseases are an emerging health challenge worldwide and include cardiovascular diseases (Ischemic heart disease, myocardial infarction), stroke, chronic respiratory diseases and diabetes. Altogether, in South-east Asia, they are accounting for $54 \%$ of annual mortality and $47 \%$ of burdening diseases (Chen et.al., 2008).

While giving the global status report on non-communicable diseases, World Health Organization (2014) indicated that, these diseases currently cause more deaths than all other causes combined. These deaths were projected to increase from 38 million in 2012 to 52 million by 2030. This report also showed that, four major NCDs (cardiovascular diseases, cancer, chronic respiratory diseases and diabetes) are responsible for $82 \%$ of NCD deaths.

In Zanzibar, the situation of diabetes has been alarming since independence of 1964. The first National NonCommunicable Disease Survey (2011) done in Zanzibar, showed that, the prevalence of Hypertension was 33\% and diabetes 3.7\%. In Zanzibar, the number of geriatric people using traditional medicine for NCDs management is considered to be increasing. However, there is no population based data to conclude the reported increment. In addition to that, the Annual Health Bulletin (2017) showed that, according to the STEP Survey done in 2011, the prevalence of diabetes was 3.7\% and diabetes was among the ten top causes of hospital admissions for all ages and ten top causes of death for Zanzibar in 2015-2017. Vogt et.al., (2017) conducted a research on assessment of diabetic polyneuropathy in Zanzibar: Comparison between traditional methods and an automated point-of-care nerve conduction device. Their study found that, mean age was 54 years, $90 \%$ had type 2 diabetes and with 9 year average disease duration. Mean HbAlc was $8.5 \%$ (69 mmol/mol), blood pressure 155/88 $\mathrm{mmHg}$. Sixty-two\% reported numbness, $61 \%$ had positive monofilament and $79 \%$ positive Sibbald tool. NCS defined neuropathy in $45 \%$ of the patients. 
Furthermore, Omar et.al., (2014) conducted a study on evaluating the effectiveness of opportunistic eye screening model for people with diabetes attending diabetes clinic at Mnazi Mmoja Hospital, Zanzibar. They found that, the targeted eye screening of diabetics showed that, 231/356 (65\%) of the subjects had eye problems, including potentially blind conditions that required immediate intervention in contrast to the existing self-reported referral where only $10 \%$ of an average of 200 diabetics underwent eye checkup in a year. This shows the extent to which this health challenge is affecting the country in general.

Several interventions have been taken by the Revolution Government of Zanzibar in order to manage this problem in the society. Among the interventions taken by the Revolution Government of Zanzibar through Ministry of Health is to establish NCD unit which was established in 2012 to oversee all NCD facilities and scale up NCD services from 8 before 2012 to 50 to date and the main objective was to see people from the community receiving proper management and the community be aware of NCDs prevention and control. This would reduce the mortality and mobility rates in the country.

The Ministry of Health Zanzibar recognizes and advocates for the use of traditional medicine and supports the idea of incorporating it into the national health system with the aim of ensuring that, traditional medicine treatment is done scientifically, rationally and with safety to the Zanzibar communities. The Zanzibar Traditional and Alternative Medicine Policy (2008) seeks to promote collaboration and partnership with all relevant partners. It also aims at promoting appropriate methods and technologies in the development of Traditional and Alternative Medicine (TAM) practices that will improve the quality, safety and efficiency of TAM products.

Therefore, the interest of the researchers was strongly motivated by the prevalence of both traditional and modern clinics used by the geriatrics in the management of diabetes as the Report from NCD Unit (2018) illustrated that, the management of diabetes to many of the patients is wanting due to the excessive usage of traditional medicine in Urban West Region of Unguja. The major objective of this study was to examine the impact of traditional and modern clinics on diabetes control in Zanzibar. The following section covers the literature reviewed and methodology employed for data collection only to end with results and conclusion.

\section{Literature Review}

\subsection{Traditional and Modern Clinic on Diabetes}

Traditional and modern clinic systems of medicine were developed using different philosophies with different cultural backgrounds. However, both of them focused at health, diseases and causes of diseases in different ways with different approaches to health and diseases. World Health Organization (2000) explains these two systems as; traditional medicine; this is the ancient and culture-bound medical practice which existed in human societies before the application of modern science to health. The practice of traditional medicine varies widely, in keeping with the societal and cultural heritage of different countries. Whereas modern medicine is the types of clinic which developed very quickly and made major contributions to disease control in the past century.

Angelantonio et.al., (2010) define diabetes as a chronic disease that occurs either when the pancreas does not produce enough insulin, when the body cannot effectively use the insulin it produces. Insulin is a hormone that regulates blood sugar. Hyperglycemia, or raised blood sugar, is a common effect of uncontrolled diabetes and over time, lead to serious damage to many of the body systems.

The use of traditional/complementary medicine around the world has increased significantly. The study conducted by Alqathama, et.al., (2020) on herbal medicine from the perspective of type II diabetic patients and physicians, they found that, $68 \%$ of the participants were frequent consumers of herbal remedies, especially cinnamon, ginger and fenugreek. In terms of patients' knowledge of herbal usage, it was found that, many (71.4\%) did not bother to consult or inform their doctors about their choice to self-medicate with herbs.

Regarding the efficacy and safety of herbal usage in diabetes, they found that, almost all the participants believed that, herbal medicine is effective $(54 \%)$ and safe $(46 \%)$ for treating symptoms of diabetes. More importantly, two-thirds of the doctors $(66 \%)$ routinely asked patients whether they applied herbs for their condition or not. Although $25 \%$ of the doctors took a positive view of herbal medicine in relation to diabetes, others expressed concerns with the rise in herb usage and wished to see more attention paid to safety aspects. This presupposed the extent to which the traditional clinics were useful to diabetic patients.

Notwithstanding, Lunyera et.al., (2016) conducted a study on traditional medicine practices among community members with diabetes mellitus in Northern Tanzania. They enrolled 481 adults of whom 45 (9.4\%) had diabetes. The prevalence of traditional medicine usage among individuals with diabetes was $77.1 \%$ (95\% CI $58.5-89.0 \%)$ and the prevalence of using traditional medicine and biomedicines concurrently was $37.6 \%(95 \%$ CI 20.5-58.4\%). Many were using traditional medicine specifically to treat diabetes (40.3\%; $95 \%$ CI 20.5-63.9) and individuals with diabetes reported seeking healthcare from traditional healers, elders, family, friends and herbal vendors. All of this literature points to one fact that, traditional clinics were commonly used by patients of diabetes.

On the other side of modern clinics, Kalra et.al., (2019) conducted a study on glucocrinology of modern sulfonylureas: this is clinical evidence and practice-based opinion from an international expert group. The study 
found that, clinical experts arrived at a consensus for the usage of modern sulfonylureas in the presence of other endocrine dysfunction and the impact of these drugs on endocrine health. It concluded with clinical expert opinion supporting the use of modern sulfonylureas in persons with diabetes mellitus.

\subsection{Diabetes Management}

Diabetes management is challenging for the patient as well as the healthcare providers. It is not always easy to keep up with exercise schedules, diabetes meal plans, blood sugar check, medications and much more, Bureau (2020). World Health Organization Report (2020) showed that, in 2014, 8.5\% of adults aged 18 years and above had diabetes. In 2016, diabetes was the direct cause of 1.6 million deaths and in 2012, high blood glucose was the cause of another 2.2 million deaths. Apparently, between 2000 and 2016, there was a 5\% increase in premature mortality from diabetes. The situation is so terrible around the world especially in developing countries. Many nations tried to find means of managing this disease. Some people prefer to use traditional medicines and other prefer modern clinics. But, all in all, whichever direction one chooses to use, the disease must effectively be managed.

Romesh (2020), in his project on Type 2 Diabetes Mellitus Treatment \& Management, argued that, the American Diabetes Association has released condensed recommendations for Standards of Medical Care in Diabetes. This means that, modern clinics are preferable for diabetes management rather than traditional clinics.

Mario (2018), argued that, modern medicines are valuable for diabetes management in America. Statistics is showing that, $57.9 \%$ of patients with diabetes have 1 or more diabetes-related complications and $14.3 \%$ have 3 or more. Strict control of glycemia within the established recommended values is the primary method for reducing the development and progression of many complications associated with microvascular effects of diabetes (for example, retinopathy, nephropathy and neuropathy).

Furthermore, a study conducted by Rose et.al., (2019) on traditional medicine and its role in the management of diabetes mellitus, found out that, 140 participants who answered the questionnaire, $67 \%$ reported using traditional medicines to manage their including $58.6 \%$ who reported using both conventional medicines and traditional medicines. Whereas some participants believe that combining conventional and traditional medicines improved the effectiveness of treatment. Apparently, the respondents gave reasons why they preferred using traditional medicines than modern medicines because of high cost of conventional treatment and the availability and accessibility of the traditional medicines. The foregoing review depicts that, still, there is a gap of what are actual impact of these methods of diabetes management (traditional and modern clinics) especially in the Zanzibar context.

\section{Methodology}

This study adopted the quantitative research design. It enabled the investigators to explore, explain a phenomenon and interpret data statistically. It also enabled the researchers to provide remedies to the challenge under investigation. For data collection purposes, an open-ended questionnaire was developed. The respondents were required to indicate how much or to what extent they felt to respond to the questions given.

Stratified random sampling was used to distribute 80 questionnaires to the respondents (geriatric patients suffering from Diabetes attending PHCU +). All questionnaires were received back. Respondents were classified by gender, age, marital status and occupation. Cronbach's Alpha test was conducted to measure the reliability of the instrument for all items and the result was 0.750 indicating a satisfactory level of internal consistency. The statistical tools used for data analysis was inferential statistics such as correlation. Data was analysed by the help of a Statistical Package for Social Sciences (SPSS) software version 23.

\section{Results and Discussion}

\subsection{Demographic Features of the Respondents}

In this study, characteristics of respondents were accorded much significance given the problem of investigation at stake. Therefore, characteristics namely, age, gender, education, working experience of the 80 respondents were examined as clearly observed in Table 1:- 
Table 1 Demographic Features of the Respondents

\begin{tabular}{|l|l|l|}
\hline Variable & Category & Percentage (\%) \\
\hline Gender & Male & 43 \\
& Female & 57 \\
\hline \multirow{4}{*}{ Age } & $61-65$ & 36.3 \\
& $66-70$ & 35 \\
& $71-75$ & 21.3 \\
& 76 -above & 7.6 \\
\hline Education & Primary & 18.8 \\
& Ordinary & 40 \\
& Advanced & 16 \\
& College/University & 5.2 \\
& Uneducated & 20 \\
\hline \multirow{3}{*}{ Occupation } & Employed & 10 \\
& Self employed & 21.3 \\
& Retired & 33.8 \\
\hline Period of living with diabetes & Unemployed & 35 \\
& 1 year below & 27.5 \\
& $1-5$ years & 47.5 \\
& 5 years and above & 25 \\
\hline
\end{tabular}

\section{Source: Researchers, 2020}

From Table 1, the number of female geriatric patients suffering from Diabetes is higher than their counterparts, the male geriatric patients. This is so due to the fact that, the population of women in Zanzibar who are suffering from Diabetes attending PHCU + is high.

Specifically, Table 1 shows that $71.3 \%$ of the respondents were between the age range of $61-70$, which is more than half of the total respondents. This means that, many geriatrics starting from 60 years are suffering from diabetes in Urban West Region of Unguja while from the age range of 76 and above, the number is low due to life expectancy in Tanzania in general because not many geriatrics are lucky enough to reach this age and that is why respondents in range (76 and above) were very few.

It is observed in Table 1 that, the patients suffering from Diabetes were employed in different fields. The largest number of patients suffering from Diabetes was unemployed and retired, which present $35 \%$ and $33.8 \%$ respectively. This means that, most of the diabetes patients who are geriatrics in Urban West Region of Unguja are not working or they are retired at the moment. This is supported by the official information that, unemployment rate in Zanzibar is $34 \%$ with a much higher real rate of unemployment and joblessness is estimated youth as high as $85 \%$.

Many of the diabetes patients who are geriatrics are educated as they attended formal education and attained various education levels and/or qualifications. As 32 equal to $40.0 \%$ attended school until Ordinary level of education. This means that, diabetes patients (geriatrics) most of them are educated hence they clearly understand diabetes as a health challenge that requires adequate attention to manage it.

Lastly, findings show that, almost half of the respondents equal to $47.5 \%$ are suffering from diabetes starting from 1-5 years. This means that, among the geriatrics, many of them are suffering from diabetes. Hence, the geriatrics need proper medical check-ups and as often as possible in order to appreciate their diabetic conditions and can live longer with the challenge.

\subsection{Correlation Statistical Techniques}

Understanding the impact of traditional and modern medicine on diabetes management is crucial. Many of modern medicines are generated from traditional medicine like plants and the Ministry Health (Zanzibar) recognizes the use of both. So, the outcome of that medicine in controlling diabetes is a priority. Table 2 is emphatical on Pearson correlation coefficient.

Table 2: Interpretation of Pearson's correlation coefficient (r)

\begin{tabular}{ll}
\hline Values of Pearson's correlation coefficient & Interpretation \\
\hline $\mathrm{r}=0.10$ to 0.29 & Small correlation \\
$\mathrm{r}=0.30$ to 0.49 & Medium correlation \\
$\mathrm{r}=0.50$ to 1.0 & Large correlation \\
\hline
\end{tabular}

Source: Cohen, $(1988, \operatorname{pg} 79-82)$

The results of the Pearson's correlation coefficient (r) as seen in Table 2, show that, all predictor variables namely; traditional and modern medicines correlate positively with the dependent variable; diabetes control. Analysis of this correlation is depicted in Table 3. 
Table 3: Correlations Analysis

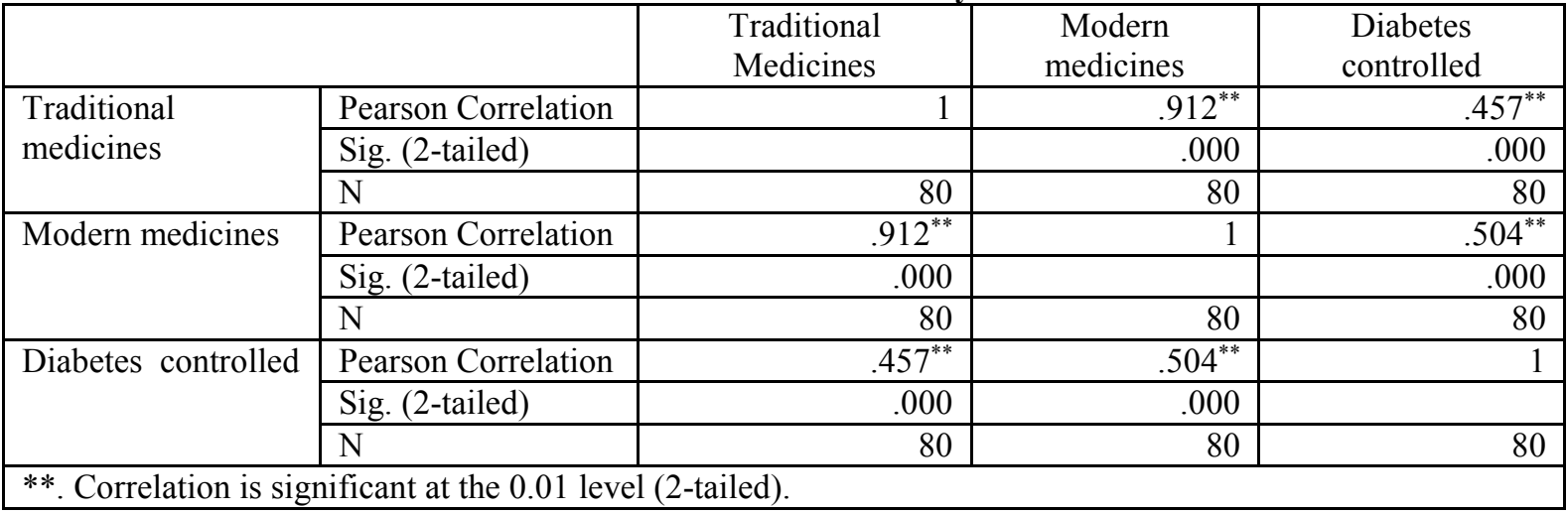

\section{Source: Field Data, (2020)}

It is observed from Table 3 that, in regard to the strength of the relationship, it is clearly seen that, independent variable (modern medicines) has greater correlation with diabetes control compared to traditional medicines. This Table indicates that, the correlation between modern medicines and diabetes control among diabetes patients in Urban West Region, Unguja is $0.504, \mathrm{p}<0.01$; two-tailed, while the Pearson's correlation between traditional medicines and diabetes control among diabetes patients in Urban West Region, Unguja is 0.457, $\mathrm{p}<0.01$; two tailed.

With reference to Cohen (1988), thresholds of interpreting the strength of the correlation regardless of the direction of the sign either positive or negative, it could be concluded that both modern medicines have large correlation with diabetes control among diabetes patients in Urban West Region, Unguja $(0.5045, \mathrm{p}<0.01)$ and traditional medicines have medium correlation with diabetes control $(0.457, \mathrm{p}<0.01)$.

Although the examination of the relationship under this objective was basically between independent and the dependent variables, it was found necessary also to report the correlations of both independent variables by them (traditional and modern medicines). As depicted in Table 3, it is seen that, both variables are highly correlated where the increase of score in one variable, it also leads to the increase of the score in the other variable $(.912, p$ $<0.01)$. This result indicates that, both medicines are useful in treating diabetes, though the findings show that, modern medicines are more effective in treating diabetes than traditional medicines.

The results of this study supported by the results of the study conducted by Rose et.al., (2019), based on Traditional Medicine and its role in the management of Diabetes Mellitus: "Patients' and Herbalists' Perspectives. Studies reported that, $80 \%$ of people in developing countries depend on traditional medicines as the primary remedy for various ailments. Worldwide, plant-based traditional medicines are the most commonly used form of treatment for a larger range of health problems. These traditional medicines play an important role in primary health care in many developing countries. Tanzania is among the countries where majority of the population depend on traditional medicines for the management of their health problems, including diabetes.

Lunyera et.al., (2016) reported that, $77 \%$ of diabetic patients in Northern Tanzania use traditional medicines for the management of diabetes. Maregesi (2016) reported that, there are various traditional medicines used by diabetic patients in Northern Tanzania, many of which have not been documented and their pharmacological properties with respect to blood glucose control have not been studied. The use of traditional medicines may be attributable to sociocultural perspectives within the community and they may contribute to disease-related complications.

Most traditional medicines are common foods which include vegetables, flowers, fruits, seeds, spices and herbs. Some foods provide health benefits beyond their nutritional value and are effective in the prevention and treatment of various diseases. This study aimed at examining the impact of traditional and modern clinics on diabetes management in Urban West Region of Unguja.

\section{Conclusion}

Findings revealed that, $72.5 \%$ of the geriatrics diabetes patients agreed that, the cooperation between traditional clinics and Ministry of Health have benefits to the society as each and everybody understands his duties for the development of this nation and conduct research on the suitable and affordable medicines for the diabetes control, treatment and overall management.

\section{Recommendations}

Basing on the results provided in this study, the researchers offer the following recommendations:-

In the first place, there is urgent need for the provision of both awareness and education concerning this killer disease; diabetes. Education should be on the prevention of the disease, risk factors of the diseases, life style 
modification for prevention and controlling the disease. Such knowledge should not only go to the geriatrics, but to the people of all generations.

Secondly, the Ministry of Health should conduct research on the common traditional medicines which are used by the diabetes patients in controlling diabetes to test if they are useful and effective for the diabetes treatment and overall management.

The cooperation which exists between traditional healers and Ministry of Education (Zanzibar) must be strengthen in order to fulfill the duties of helping the people of this Region and Nation in general for their sustainable wellbeing.

\section{References}

Alqathama, A., Alluhiabi, G., Baghdadi, H. et al. (2020) Herbal medicine from the perspective of type II diabetic patients and physicians: what is the relationship?. BMC Complement Med Ther 20, 65 https://doi.org/10.1186/s12906-020-2854-4

Asefa, A., Zewudie, A., Henok, A., Mamo, Y., \& Nigussie, T. (2020). Depression and Its Associated Factors among Diabetes Mellitus Patients Attending Selected Hospitals in Southwest Ethiopia: A Cross-Sectional Study. Psychiatry Journal

Bureau, N. (2020). The Impact on Diabetes Management \& Future Considerations. Express Healthcare,

Kalra, S., Das, A.K., Baruah, M.P. et al. (2019). Glucocrinology of Modern Sulfonylureas: Clinical Evidence and Practice-Based Opinion from an International Expert Group. Diabetes Ther 10, 1577-1593 https://doi.org/10.1007/s13300-019-0651-1

Lunyera, J., Wang, D., Maro, V. et al. (2016) Traditional medicine practices among community members with diabetes mellitus in Northern Tanzania: an ethnomedical survey. BMC Complement Altern Med 16, 282 https://doi.org/10.1186/s12906-016-1262-2

Maregesi, S. M., Kauke, B., Kagashe, G., \& Kaali, R. (2016). Traditional eye medicines in Tanzania: Products, health risk awareness and safety evaluation. Herb. Med. Open Access, 2(1).

Mario, S. (2018). Diabetes Mellitus Treatment. The Cleveland Clinic Foundation

Nzali, A. S. (2016). Determinants of access to free health services by the elderly in Iringa and Makete Districts, Tanzania (Doctoral dissertation, Sokoine University of Agriculture)

Roglic, G. (2016). WHO Global report on diabetes: A summary. International Journal of Noncommunicable Diseases, 1(1), 3.

Romesh, K. (2020) Type 2 Diabetes Mellitus Treatment \& Management. Medscape. https://emedicine.medscape.com/article/117853-treatment

Rose, K., Haikael D. M., and Judith K. (2019). Traditional Medicine and Its Role in the Management of Diabetes Mellitus:(Patients' and Herbalists' Perspectives. Hindawi Evidence-Based Complementary and Alternative Medicine

Stephanie W. (2020): Everything You Need to Know About Diabetes. Healthline. https://www.healthline.com/health/diabetes

Teshome, H. M., Ayalew, G. D., Shiferaw, F. W., Leshargie, C. T., \&Boneya, D. J. (2018). The prevalence of depression among diabetic patients in Ethiopia: a systematic review and meta-analysis, 2018. Depression research and treatment, 2018

The International Diabetes Federation (IDF) (2020) COVID-19 and diabetes. https://www.idf.org/aboutdiabetes/what-is-diabetes.html

\section{Biodata of the author}

Zuhura Saleh Amour, born 1968 in Zanzibar. She promoted to current position as a senior officer at Non Communicable Diseases Unit Ministry of Health Zanzibar. Befor that she was taking a several positions such as; Senior Nurse Midwife -General Pediatric ward Chake Chake Hospital Pemba, Assistant In charge Intensive Care Mnazi Mmoja Hospital Zanzibar, Incharge female General Ward and head of Maternity Ward Mnazi Mmoja Hospital Zanzibar and Human Resource officer Mnazi Mmoja Hospital Zanzibar. She graduated at Kampala International University- Dar-es -salaam (2010) with Postgraduate Diploma in Human Resource Management Zanzibar. Master in Public Administration at Zanzibar University (2020). She also participated in different NCDs training as a participant and facilitator. In addition, she did advocacy to promote adherence to the WHO framework convention on tobacco control. Finally, she is a member of Zanzibar Nurses Association, Zanzibar Non Communicable Diseases Alliance since 2012 and Zanzibar Cancer Association since 2012. 